\title{
Narrative review of home care for palliative patients in general practice
}

\author{
Lyubima Despotova-Toleva ${ }^{1}$, Nina Toleva-Nowak ${ }^{2}$ \\ ${ }^{1}$ Department of Urology and General Practice, Medical University, Plovdiv, Bulgaria; ${ }^{2}$ Department of History and Theory or Architecture, UACEG, \\ Sofia, Bulgaria \\ Contributions: (I) Conception and design: L Despotova-Toleva; (II) Administrative support: N Toleva-Nowak; (III) Provision of study materials or \\ patients: Both authors; (IV) Collection and assembly of data: L Despotova-Toleva; (V) Data analysis and interpretation: L Despotova-Toleva; (VI) \\ Manuscript writing: Both authors; (VII) Final approval of manuscript: Both authors. \\ Correspondence to: Lyubima Despotova-Toleva, MD, PhD. 29 Prezviter Kozma Str., Plovdiv 4000, Bulgaria. Email: desptol@abv.bg.
}

\begin{abstract}
In 2019, the International Association for Hospice and Palliative Care (IAHPC) presented for adoption and signing some very important documents, among which "New definition for Palliative Care", "Letter for National Palliative Care Advocates" (which was designated to be sent to government lead on Universal Health coverage, and UN negotiations) and "Suggested language changes to zero draft 'Political Declaration of the High-level Meeting on Universal Health Coverage" - "Universal Health Coverage: Moving Together to Build a Healthier World". They are devoted to the constantly growing need of palliative care in addition to the diagnostics and treatment, and are essential part of the care both for the patients with chronic incurable life-threatening diseases (especially with serious health-related suffering) and their families. As the home is the natural place of illness, general practitioners (GPs) should be prepared and involved in the palliative care spectrum of activities. The authors present some aspects of the home-based care while focusing on some problems and challenges, and making comparison with some specific issues for palliative home-care. The overview of the new policies and documents for palliative care reveals the ultimate importance of "universal access to high-quality palliative care, integrated into all levels of health care systems in a continuum of care with disease prevention, early diagnosis, and treatment, to assure that any patient's or family caregiver's suffering is relieved to the greatest extent possible" and the key role of GPs in it.
\end{abstract}

Keywords: Palliative care; general practitioner (GP); home-based care; telemedicine; interdisciplinarity

Submitted Mar 23, 2020. Accepted for publication May 10, 2021.

doi: 10.21037/apm-2021-01

View this article at: http://dx.doi.org/10.21037/apm-2021-01

"General practice is the easiest job in the world to do badly, but the most difficult to do well." - Prof. Sir Denis Pereira Gray

\section{Introduction: the growing need of palliative care}

The need of palliative care is constantly growing worldwide and the spectrum of patients and diseases, leading to the need of palliative care is very broad (1). According to recently published data in the "Letter for National Palliative Care Advocates to send to government lead on Universal Health coverage, and UN negotiations" (2) by 2060, an estimated 48 million people ( $47 \%$ of all deaths globally) will die each year with serious health-related suffering, and $83 \%$ of these deaths will occur in low-income and middleincome countries.

Due to the phenomenon of the so-called "silver tsunami" (the increasing number of elderly, and those of them with severe, incurable, life-limiting and life-threatening diseases) the problem escalates dramatically. Thus, significant and urgent changes should be made in some basic documents, including "Suggested language changes to zero draft 'Political Declaration of the High-level Meeting on Universal Health Coverage" - "Universal Health Coverage: Moving Together to Build a Healthier World" (3). 
A number of international organizations have been working successfully for years to expand the access and to improve the quality of continuous and palliative care. There are a number of Standards developed (4-6), as well as a vast amount of other documents such as strategies (7), national consensuses (8), programs (9), models $(10,11)$, frameworks (12), guidelines and protocols (13). All these documents have been developed both for adult patients and children, and are being constantly elaborated and improved. Yet, currently in Bulgaria, there is no palliative care standard, clinical guideline or strategy. There is no palliative care policy/ health policy and the only way for patients to receive palliative care is the clinical pathway, preserved for $4^{\text {th }}$ stage cancer patients.

The Global Atlas of Palliative Care at the End of Life (14) put Bulgaria in Group 3a-defining it as a state with isolated palliative care provision. Earlier study "International Observatory on End of Life Care: A Global View of Palliative Care Development" (15) also has determined Bulgaria in Group 3 with localized provision of palliative care. Even though there are researched, stressing on the role and importance of research in promoting palliative care practices (16), the opportunities for long-term and palliative care are too limited. There are no trained specialists (doctors, nurses, non-medical specialists), there is no recognized specialty within which to train the much-needed specialists and teams. There is no state-level standards for the provision of continuous and palliative care for children and adults. There are no structures (hospital wards, day hospitals, long-term and palliative care offices, clinics and offices for pain treatment, mobile teams, home palliative care, etc.) in place to provide qualified palliative care There are some private hospices available only for patients who can afford the high costs. Most of the time, Bulgarian GPs cannot fully rely on the hospice care and the hospice personnel, as they are also not trained in providing longterm and palliative care.

A major problem is that the hospices in Bulgaria which are registered in accordance to the law for medical establishments/institutions are few. In 2019, architect Nina Toleva-Nowak made an overview of the availability and the distribution of registered hospices in Bulgaria and gathered the result in an interactive map, including information about each facility.

Her study showed that there are overall 50 registered hospices in Bulgaria, 30\% of which are located in the capital city-Sofia. Another bothering signal was that in 10 out of
28 regions, there are no hospices at all. In other 9 regions there is only one hospice facility. In 5 regions, 2 hospices per region, only in 2 regions there were 3 hospices and in another two-Sofia-city and Varna-more than three hospices, mostly located in the main city for the region. Another major problem is that many facilities for elderly are announced as hospices, yet they are not registered in accordance to the law and are not equipped with qualified and trained personnel. Thus, the majority of the patients with palliative care needs rely on almost entirely on their general practitioners (GPs), who send them in case of emergency for hospitalization.

On the other hand, in 2019 the IAHPC presented for adoption and signing some very important documents, among which: "New definition for Palliative Care", "Letter for National Palliative Care Advocates to send to government lead on Universal Health coverage, and UN negotiations", and "Suggested language changes to zero draft 'Political Declaration of the High-level Meeting on Universal Health Coverage" - "Universal Health Coverage: Moving Together to Build a Healthier World" (17). They are devoted to the constantly growing need of palliative care in addition to the diagnostics and treatment as an essential part of the care both for patients with chronic incurable life-threatening diseases (especially those with serious health related suffering) and for their families. An important aspect, is that the patients have the right to receive the necessary palliative care at a place of their choice. This led to the fact that a considerable part of patients wish to receive care at home or at the home of their loved ones and to be monitored by a physician they trust. As home is the natural place of illness, GPs are the most suitable medical specialists to provide and/or lead home-based palliative care and thus they must be prepared and involved in palliative care spectrum of activities. As a family physician (FP) the GP is in better position to provide this type of care, centered to the patients and their families (18), according to the new 2019 IAHPC definition (19). Another valuable aspect is noted by R. Steele and B. Davies-the dimensions of family functioning: given examples of the range of behaviors, distinguishing more helpful from less helpful when integrating the past, dealing with feelings, solving problems, utilizing resources, etc. (20).

We present the following article in accordance with the Narrative Review reporting checklist (available at http:// dx.doi.org/10.21037/apm-2021-01). 


\section{Objectives}

The aim of our study is contribute to the understanding of the importance of home care for palliative care patients through analyzing and presenting scientific publications in several important sub-topics in homecare for palliative care patients and to enhance the awareness and the knowledge of the GPs in providing better home care for this vulnerable group.

\section{Methods}

Literature review made by two independent researchers through PubMed search, EMBASE and free search through combinations of key words (incl. palliative care, home care, general practice, GP, barriers, place of death, telemedicine, etc.), Publications were selected regardless of language of publication, year of publication The initial search and selection of scientific publications for the purposes of the review was carried out as follows-after the initial selection by keywords, the found materials were sifted by titles, then selected publications were reviewed by abstracts, of which after careful selection and overlapping results from the two researchers were selected full-text articles. For the final stage were selected the most appropriate ones.

\section{Discussion}

The importance of home-based care is proven by many studies. Published systematic reviews and original articles focus on the preferences of palliative patients to stay at home and to die there, surrounded by relatives and friends and attended by their GP (21-23).

As the home-based palliative care becomes a part of home-based medical practice in different primary care practices, more studies have assessed the benefits and challenges, including the organization of a palliative care team, the patient characteristics, the use of electronic medical records, systems and technology and the urgent care delivery when necessary. Norman et al. also stress on community-oriented component of care (24).

Providing patients and families with quality palliative care at home is a part of the comprehensive primary care. The holistic approach, used in general practice helps immensely to deal with the complexity of palliative care patient problems, yet the GPs still report a lack of confidence as feel not trained enough to deal with such issues and consider the available resources insufficient. The poor communication between GPs/FP and other specialists and treating teams are recognized as barriers to provide comprehensive palliative care. Despite these difficulties and barriers, they are ready to help the patients and to improve their own knowledge and skills in the field of palliative medicine (25).

Of particular importance for patients with cancer at the last stage is to receive quality palliative care and management of severe concomitant symptoms, tailored to their wishes, preferences and expectations, but unfortunately in many countries, including Bulgaria, this cannot be achieved due to lack of awareness, regulations, properly trained staff etc. Often GPs have to act as mediators between patients and oncologists (26) and to become advocates of the patients and their families against the healthcare system and the community and to facilitate the relations with hospital specialists to ensure continuity of care. Having that in mind, the home-based palliative care could bridge the gap especially "for patients who require intensive home management, but are not enrolled in hospice" (27).

Peng et al. have studied the willingness of GPs and some rarely explored factors influencing the palliative care at home. Usually the FPs tend to "limit their services to and referral and were less likely to see patients and prescribe medicine, to provide phone follow-ups, to provide home visits or to offer bereavement care for the family" (28). Interestingly, a prior Taiwan study revealed low willingness of FPs to provide palliative home care (29).

In recent years, the need of palliative care programs to enhance patient wellbeing and improved outcome is increasing. For the patients, their families and the society it could be achieved by home-based palliative care, moreover at cost, lower than other healthcare options (30).

Different tools could help GPs better the symptom management to asses provided care and also to predict survival time for patients on home-base palliative care $(31,32)$.

A number of protocols are used to improve home palliative care and to stimulate and promote the use of multidisciplinary teams to cover better the needs of the palliative patients and their families. Some of them are specially designed to cover specific problems, diseases or symptoms in palliative patients and their relatives (33-35).

In GP's work with palliative patients, the specialized knowledge and skills of other healthcare professionals is needed. It is believed that he should be the leader of the interdisciplinary palliative care team in general 
practice, with a pivot role ate decision-making process, while coordinating and controlling the care dynamics in accordance to the changing needs of patients and their relatives (36).

A study by Johnson et al. has recognized the GPs as the key professionals in linking people with specialist palliative care $(37,38)$.

Another study by Danielsen et al. demonstrates that "optimum palliative care at home depends on close collaboration and dialogue between the patient, family, home care nurses and general practitioner" (39).

Other benefits of home palliative care include reduced emergency department visits and hospitalizations rates and increasing patient and caregiver satisfaction, while reducing the morbidity and mortality associated with the avoidable hospitalizations. Moreover benefits are proven (40).

By maintaining continuing relationships with patients and families, based on trust and solidarity, the GP becomes the key person in a cost-effective healthcare systems and ensures better health outcomes (41).

\section{Palliative care for migrants in general practice}

Recently, with the increasing flow-dynamics of migrants, new and unknown challenges are emerging for GPs, for which they need to be prepared (42).

Migrant-specific aspects of the palliative care put additional stress to GPs (verbal and non-verbal communication; language barriers especially in the process of decision-making; dealing with patient preferences, which may conflict with his professional values; dealing with cultural issues and the ensuing conflicts between patients, families and professionals; difficulties in the access to healthcare system). Here is to mention the importance having intercultural teams which apply holistic approach in order to help to overcome these specific difficulties and to proper organize and plan the needed care. As conflicts between patients, families and professionals may occur, the cultural competence and multicultural teams could alleviate and better the care management (43). GPs need more training and understanding in cross-cultural communication and consultations, thus enhancing the understanding and the trust between them and migrant patients. Other obstacles for migrants to receive proper palliative care are related to the diversity aspects (psychosocial, sex and socio-economic status). Overcoming language barriers is crucial in regard to patients' rights and when achieving informed consent is needed.
Systematic review of 30 articles pointed out three main themes: "palliative care practice within the family, trust as a precondition of palliative care, and the importance of knowledge and cultural competency" (44-56).

\section{Barriers to bome palliative care}

Barriers to home palliative care are studied from different perspectives. An analysis of the barriers to needs growth in home palliative care has presented some major challenges such as "a lack of clarity on the applicability of national standards to home-based programs, a lack of sustainable financing methods for home-based palliative care, and lack of awareness of existing support resources for program design and leadership development" (57-59).

Usually the GP is the leader of the palliative care team for home-care and specialists are selected as team members according to palliative patient condition and needs. To meet the complex needs of the palliative patients and to deliver holistic end-of-life care to patients and their families, an inter-professional team approach is recommended. The teams are supposed to help and support the FPs-a practice explored in a Canadian study focusing on "institutionrelated barriers (i.e., the healthcare system), interest-related barriers (i.e., motivations of stakeholders) and Idea-related barriers (i.e., values of stakeholders and information/ research), on sociopolitical barriers" (60). Research on barriers in palliative care has found that patient well-being is improved by palliative teams where psychologists and psychiatrists are core members of the treatment team (61). That is why integrated care teams are needed to ensure high quality palliative care at home.

The main goals of home palliative care include reducing suffering and enhancing quality of life, especially for paediatric patients and their parents. That is why recognizing potential team level inhibitors could contribute to better care models. Walter et al. have found 8 inhibitors to team level regoaling, among which team challenges due to hierarchy, avoidance of criticizing colleagues, structural communication challenges, group norms in favor inhibited expression of sorrow, lack of social support, reinforcement of labeling and conflict (62). As Interpersonal aspect of care could be source of barriers, the trust and confidentiality between the patient and the GP are fundamental factors in general practice-the patients and families need physicians who are accessible and can be trusted (63).

Having not enough resources devoted to home-based care for palliative patients is a significant barrier, so there is 
a recognized need to have more resources $(64,65)$.

\section{Education in palliative care for GPs}

European Academy of Teachers in General Practice has published educational agenda focusing on primary care management, person centeredness, specific problemsolving skills, comprehensive approach, community orientation, holistic approach, central application features, integration and implementation. All of them are very important and needed when caring for palliative patients and their families (66). The education and training of GPs for the needs of working with palliative patients and their families in addition to their core competencies is aimed at acquiring specific knowledge and skills through purposeful training (67). GPs also recognize the need of improving their education and training at all educational levels (undergraduate and postgraduate level) and to be focused on specific problems and difficulties they face while carrying for palliative patients, or problems related to pain-management and dealing with other symptoms, or decreased access to hospice beds for their patients and support services. GPs declare the need of further training for recognizing the barriers to teamwork and communication. Home-based general practice is considered as appropriate teaching site for palliative care (68).

The educational paradigm using the palliative patient as unusual but successful teacher could significantly contribute to enhance quality of education for medical students and doctors, giving them another point of view on personal experience with these diseases and conditions, experience they do not have (69). It could be also part of patient empowerment strategies-involving the patients as a teacher reinforcing the patient-centered approach. Patients have important unique contribution to the training of general practice residents and help them to develop a range of competences $(70,71)$. This type of "co-teaching"-the patient and the university teacher in tandem-is valued by clinicians. It could also bring meaning in the life of palliative patients. Studies suggest continuing education is needed for GPs to provide better care for their palliative patients and families, including bereavement care (72). General guidelines about teaching palliative care incl. at home, find it excellent for training and comprehension of palliative medicine, patients and family needs and contributes to humanizing effect on trainees to improve the palliative care understanding (73). The program used experiential learning techniques, such as a clinical attachment and mentoring the education and the skills of GPs in Western Australia and the quality of care they provide, within a 14-week training program (74-77).

Meanwhile, the Ministry of Health in Bulgaria has published a National Program for GPs education. The author develops further some very important question with regard to palliative care in general practice and since 2003 is leading forty hours postgraduate courses in Palliative Medicine at the Medical University, Plovdiv. The fact is, this course is usually attended by medical doctors with one or more acknowledged specialties (anaesthesiology, internal diseases, oncology, etc.), but not by GPs. During the main one-month mandatory theoretical course the topic has been taught focusing of essentials of palliative care in one day. The GPs may also select a thesis in the field. After that they are more aware about the problems of the palliative patients and their families. A long way is ahead to accredit Palliative medicine as clinical and scientific specialty, but the most important issues are ready now: monographs in paediatric palliative care (78) and Fundamentals of Palliative medicine (79-84), a manual in palliative care (85), educational program for all levels-bachelor, master, $\mathrm{PhD}$ and project for national Palliative Care Standard (86). It is important to change the attitudes of the GPs and the society towards palliative care.

\section{The role of GPs in provision of palliative care in rural areas}

In many countries, especially in the groups with no or isolated palliative care facilities, the GPs, face significant difficulties in providing care for such patients and their families and in managing the symptoms and the situations. Comparing the hospice map for Bulgaria (Figure 1) with the regional distribution of GPs, it became obvious, that the regions with lower number of GPs are also lacking hospices. This, along with the decreasing number of GPs over the years (last data from 2019 showed that there are 4,176 GPs in Bulgaria) and with the increase in their average age (approximately 60 years average age of Bulgarian GPs with on average 6 months difference between females and males), make the process particularly challenging. In many countries rural GPs are heavily involved in looking after palliative care patients. They recognize the need for nursing and medical palliative care consultant services to be available as well as they require undergraduate, postgraduate and continuing education (87).

A systematic review of twenty-six studies stated that 


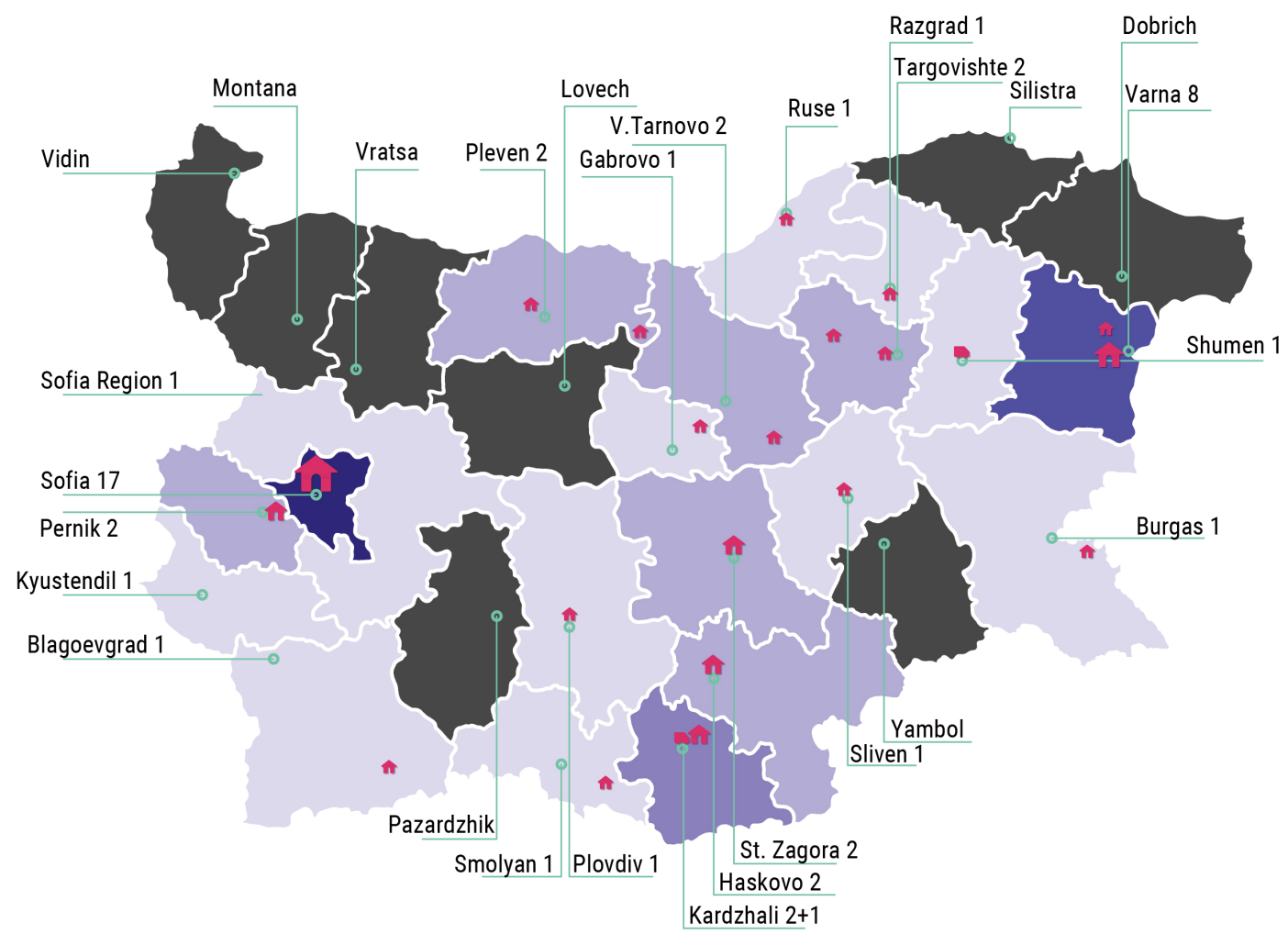

Figure 1 Google-based map of registered hospices in Bulgaria, architect Nina Toleva-Nowak.

palliative care services have developed mostly in urban areas, while rural areas typically experience lack of wellorganized services, so GPs and nurses undertake the most of palliative care. Hope for solving some of the problems are given to the improvement and application of modern ICT and telemedical activities (88). Including telemedicine with appropriate training, especially in areas with shortage of GPs could contribute to better palliative care in rural areas. There is a marked need to support rural GPs (89) using different medical and nonmedical resources, including contacting and involving local authorities and community leaders when working in remote/rural areas (90). GPs can partially cover palliative care needs of their patients and families at home (point of care-home!), but they need not only support from other professionals, but also external recourses, effective networking for clinical, educational and research purposes adapted to the specific rural conditions and to fill the gaps in service provision for children and adult palliative care (91) and to ensure "universal access to high-quality palliative care, integrated into all levels of health care systems in a continuum of care with disease prevention, early diagnosis, and treatment, to assure that any patient's or family caregiver's suffering is relieved to the greatest extent possible" (92).

\section{Place of death}

Death at home is considered as golden standard (93-95). Being the closest, longest-lasting and trusting relationship with his patients, the GP should know his patients' attitudes and wishes about their preferred place of care and place of death. Study about GPs awareness of preferred place of death found $88 \%$ of the patients prefer to die in a private or care home, while GPs were informed by the patients themselves in $84 \%$ of cases. These results are very important for the general practice (96). One of the indicators of quality of care is that the patient can die at his or her chosen place, and this is where the GP plays a key role in obtaining this information from the patient and in honoring his or her preference. It is proven that if GPs are informed, patients often die at their preferred location. GP awareness in this matter is very important $(97,98)$.

In the provision of palliative care, the dying of the patient in a chosen place is associated with quality care. GPs usually 
know the place of death of their patients, but are often unaware of their pre-selected place of death. It is therefore advisable GPs to receive such information not only from the patient himself, but also if possible, from the family members, although talking about this very sensitive topic is a challenge for him (99). When GPs provide palliative care for their patients, it positively contributes to fulfilling their desire for a chosen place of dying or dying at home (100).

Study from four European countries also was focused on desired place of death and the role of GPs. The result support those from other studies in the field, stating "despite the importance of being able to die in a preferred location, GPs were often unaware about patient preferences, especially in Italy and Spain. If GPs were informed, the preference was often met in all countries, indicating room for improvement in end-of-life care" (101). Older adults also prefer to have care and die at home (102). Patient preferences for PPOC and PPOD vary according to their diagnoses; tailoring palliative needs to patients' preferences are important regardless of their diagnosis (103).

For rural areas home is the preferred place of death (104). As compared to cities, in many rural areas there is a tendency for home death. The preferences of palliative patients are not absolute, so more research is needed do define the influencing factors (104). Research on differences in care and the preferred place of death between cities and rural areas should contribute to policy-making and strategies and help to "improve equity in health care delivery for those approaching the end of life" (105). Urban-rural differences are registered also in a Chinese study, suggesting "bridging the gaps between urban and rural areas is necessary for the reform and construction of health care and long-term care systems in China" (106).

GPs, applying preferred by patients holistic approach are becoming more and more important in providing optimal end of life care. Proactive identification of people approaching end of life is contributing to improvement of all aspects of care (107). It could be interesting to compare different countries data between them and with the statistical results from Norway (108). GPs as well as medical students and junior hospital doctors are adequately trained for their important role in caring for dying patients (109). Thus, more research on the role of GPs in comprehensive home care and end of life care is ultimately required.

\section{Telemedicine for bome-based palliative care}

The development of information and communication technologies significantly contribute to improving the diagnosis, treatment and quality of care in all areas of medicine. Telemedicine has become an useful tool and offers many benefits in the palliative care provided at the patient's home, for the GP, for the palliative team, etc. New and new telemedicine initiatives, activities and applications are developed, targeting not only medical professionals and services but also patients and their families and relatives and carers. As previously noted in this paper, GPs report need of more education and training in palliative care, so for them the availability of suitable and tailored to their needs palliative care information, including cancer care (110).

CareSearch project in its special GP section was among the activities, especially devoted to GPs to facilitate information search in the field of palliative care and to provide them with knowledge, skills and practical advice in the field. Using real time video consultations in home-based palliative care has both advantages and disadvantages. Video consultations are considered feasible and could facilitate the work of the interdisciplinary palliative team, increase the quality of specialized home palliative care and provide such care over distance. An analysis of the suitability of 'videovisits' for palliative home care: implications for practice has revealed four factors influencing the eligibility and decisions about patients' "suitability for video-visits: diagnosis, low Edmonton Symptom Assessment System score, no care-giver present, number and types of interventions required" (111). Videoconferencing is well accepted also by patients regardless of the stage of their life-illness and they tend to take an active role, while the relatives are also actively involved as well as the communication between patients, relatives and palliative care team is facilitated and strengthened. Videoconferencing is used for interactive cases discussions, consultations, assessments, training and education for specialists. The technology is usable and acceptable both for patients and health professionals (112).

One of the first one of the first randomized controlled trials in palliative telecare on effectiveness of weekly teleconsultation in complex palliative home care was performed with support of GPs (113). Telecare is rapidly growing practice in palliative care, where the communication is of high importance and understanding of the use of modern telecare in many aspects should be understood. Even face-to-face communication is essential, modern telecommunication is helpful both for patients and doctors and they are generally positive, although researches have noticed some reservations and prerequisites, in particular strong reservations are voiced against permanent 
telemonitoring in the patient's home. The importance of timely and accessible care for clinically vulnerable, dying cancer patients and their family caregivers was confirmed by Stern et al. (114).

Interesting and results are reported by Hoek et al. They discover that "adding weekly teleconsultations to usual palliative care leads to worse reported symptom scores among home-dwelling patients with advanced cancer" and offer possible explanations about this finding (115). Being one of the main features of general practice, patientcentered communication between patients and GPs is affected by the use of ICT. Study published in $2015^{\text {th }}$ concludes teleconsultation fits the practice of home-based palliative care, pointing out the patient involvement and empathic patient-professionals relationships and proposed the use of "teleconsultation implementation guide for optimal use of teleconsultation in daily palliative care practice". Children are very specific and difficult patients GPs, especially those with palliative care needs. For them according to Bradford et al. palliative tele consultations are feasible and acceptable, can reduce the burden on families at a distressing time and "are just as effective as face-toface consultations in terms of the documented components of the consultation". Web-based videoconferencing shows advantages in rural palliative care as "acceptable, effective, feasible, and efficient way to provide timely support to elderly rural patients and their families" (116).

Pinto et al. have analyzed forty mobile apps (117). Among main issues to which apps are devoted, are: communication between patients, families and palliative care teams, reinforces partnership, decreases the burden on families, use of the emergency services. drugs, tools/clinical guidelines, hospice, symptom management and palliative care information. Development of such technologies are considered good strategy, but being in the beginning, further research is needed to promote evidence-based practice. Another study has identified forty-six palliative care applications targeted to clinicians. The evidence upon which the apps were based still remains unproven (118). Mobile applications could bring additional resources to support patients and the palliative cancer care program (119). Mobile phone use is increasing also in palliative care and could contribute to better care, especially in remote areas, but some factors should be kept in mind as access to phones and ability to use smartphones, mobile network access, and limited access to expertise and hardware required for mobile health (120). Mob apps used for paediatric palliative care appear to be inexpensive and user-friendly technology, which allows to make evidence-based and informed decisions about referrals and "has the potential to be an important tool in improving health care service delivery for children". Development of apps may be expanded to other areas of child care, especially for facilitating general practice (121).

Usually m-Health apps were focus on biological and clinical monitoring (particularly concerning the symptoms), disease self-management, and therapeutic patient education. Future attention and research are needed to define which health-care professional would be in charge of this "m-monitoring" (122). There is a lot of enthusiasm towards use of mHealth to improve care coordination, facilitate communication, enhance symptom monitoring, and improve patient-family support, but there are some reservations related to depersonalized assessment and care. The use of mHealth could be and useful tool, but cannot replace important multidisciplinary services (123). An Iranian mobile health application has a lot of functionalities and could help in self-care of patients with needed to palliative care (124).

Digital health platform supports clinical decision making in opioid prescription. It is safe, reliable and validated CDS that has potential to reduce harms from opioid dosing errors (125). The University of Kansas Medical Center in partnership with Hospice Services has developed telehospice service. Among different facilities, a secure cloud-based videoconferencing solution was used. According to authors, "TeleHospice's potential to supplement and improve hospice services while reducing costs is significant, but continued research is needed to understand best fit within frontier hospices, to inform future urban applications, and to address reimbursement" (126).

In recent years, studies have emerged on some other interesting aspects of use of mobile applications in palliative patients. GPs should be aware of these new possibility to positively affect their patients status. A prototype of mobile application monitors the patients' comfort and is considered as simple, useful, feasible and user-friendly. When using mobile health applications, ensuring data security is a priority. Data protection should be carefully studied further when developing such applications (127). Weekly et al. have reviewed different mobile apps for Calming, Relaxation, and Mindfulness Interventions for Pediatric Palliative Care Patients as a relaxation and distraction tool (128).

Palliative care is also associated with significant burden on the medical and non-medical staff providing care and support, emotional and physical exhaustion/compassion fatigue and burn out syndrome may develop. There are 
various strategies to cope with the stress, incl. using mobile applications. Meditation, mediated by mobile application could help caregivers to alleviate work-related stress (129). Electronic Palliative Care Coordination Systems used by different organizations as a tool for sharing information could be used also to assess whether there was a measurable benefit with patients dying at their preferred place of death (130). Newly appeared review article reveals the need of future work is needed to evaluate the use of telehealth in palliative care and improvement of telehealth design in line with digital service standards (131-139).

\section{Summary}

Caring for the palliative patients and their families require a multidisciplinary approach and multi-institutional commitment, a high degree of responsibility, clearly formulated and distributed tasks and professional knowledge and skills for those involved in these activities.

Care for palliative patients must be harmonized with international and national regulatory base.

\section{Acknowledgments}

Funding: None.

\section{Footnote}

Provenance and Peer Review: This article was commissioned by the Editorial Office, Annals of Palliative Medicine for the series "Narrative \& Evidence-based Medicine for Traditional Medicine: from basic research to clinical practice and trial". The article has undergone external peer review.

Reporting Checklist: The authors have completed the Narrative Review reporting checklist. Available at http:// dx.doi.org/10.21037/apm-2021-01

Conflicts of Interest: Both authors have completed the ICMJE uniform disclosure form (available at http://dx.doi. org/10.21037/apm-2021-01). The series "Narrative \& Evidence-based Medicine for Traditional Medicine: from basic research to clinical practice and trial" was commissioned by the editorial office without any funding or sponsorship. LDT served as the unpaid Guest Editor of the series. The authors have no other conflicts of interest to declare.
Ethical Statement: The authors are accountable for all aspects of the work in ensuring that questions related to the accuracy or integrity of any part of the work are appropriately investigated and resolved.

Open Access Statement: This is an Open Access article distributed in accordance with the Creative Commons Attribution-NonCommercial-NoDerivs 4.0 International License (CC BY-NC-ND 4.0), which permits the noncommercial replication and distribution of the article with the strict proviso that no changes or edits are made and the original work is properly cited (including links to both the formal publication through the relevant DOI and the license). See: https://creativecommons.org/licenses/by-nc-nd/4.0/.

\section{References}

1. Worldwide Palliative Care Alliance, World Health Organization. Global atlas of palliative care at the end of life. London: Worldwide Palliative Care Alliance 2014. Available online: https://www.who.int/nmh/Global_Atlas_ of_Palliative_Care.pdf

2. Calling national palliative care leads to strengthen palliative care language on UHC negotiations [Internet]. International Children's Palliative Care Network; 2019 [cited 2020 Mar 12]. Available online: http://www. icpcn.org/calling-national-palliative-care-leads-tostrengthen-palliative-care-language-on-uhc-negotiations2/?fbclid=IwAR3a1APSsY_tfZqJ4M6JSiLhGkwcCss0s_ kAalwaC3Zi4TZaNpkQOooipYk

3. Political declaration of the high-level meeting on universal health coverage [Internet]. A/RES/74/2 - E - A/RES/74/2. United Nations General Assembly; 2019 [cited 2020 Mar 12]. Available online: https://undocs.org/en/A/RES/74/2

4. National Palliative Care Standard. 5th Edition - 2018 [Internet]. PalliativeCare-National-Standards-2018_Novweb.pdf. Palliative Care Australia; 2018 [cited 2020 Mar 12]. Available online: https://palliativecare.org.au/wpcontent/uploads/dlm_uploads/2018/11/PalliativeCareNational-Standards-2018_Nov-web.pdf

5. APPENDIX 3: The 13 standards for providing quality palliative care for all Australians [Internet]. Home Parliament of Australia. Parliament of Australia; 2015 [cited 2020 Feb 2]. Available online: https://www.aph. gov.au/Parliamentary_Business/Committees/Senate/ Community_Affairs/Completed_inquiries/2010-13/ palliativecare/report/e03 
6. Standards for Palliative Care 2019 [Internet]. HNZstandards-2019.pdf. Hospice New Zealand; 2018 [cited 2020 Feb 6]. Available online: https://www.hqsc.govt. nz/assets/Consumer-Engagement/Resources/HNZstandards-2019.pdf

7. National Palliative Care Strategy 2018 [Internet]. nationalpalliative-care-strategy-2018.pdf. Australian Government, Department of Health; 2018 [cited 2020 Feb 4]. Available online: https://www.health.gov.au/sites/default/files/ national-palliative-care-strategy-2018.pdf

8. Clinical Practice Guidelines for Quality Palliative Care, Third Edition [Internet]. nationalcoalitionhpc.org. National Consensus Project for Quality Palliative Care; 2017 [cited 2020 Feb 6]. Available online: https://www. nationalcoalitionhpc.org/wp-content/uploads/2017/04/ NCP_Clinical_Practice_Guidelines_3rd_Edition.pdf

9. National Health Mission [Internet]. National Programme for Palliative care (NPPC). Ministry of Health and Family Welfare, Government of India; [cited 2020 Mar 2]. Available online: https://nhm.gov.in/index1.php?lang=1\&l evel $=2 \&$ sublinkid $=1047 \&$ lid $=609$

10. Wiencek C, Coyne P. Palliative care delivery models. Semin Oncol Nurs 2014;30:227-33.

11. Labson MC, Sacco MM, Weissman DE, et al. Innovative models of home-based palliative care. Cleve Clin J Med 2013;80 Suppl 1:eS30-5.

12. End of Life and Palliative Care Framework 2019-2024 [Internet]. eol-pc-framework.pdf. NSW Ministry of Health; 2019 [cited 2020 Feb 20]. Available online: https:// www.health.nsw.gov.au/palliativecare/Publications/eol-pcframework.pdf

13. Standards, clinical guidelines and protocols [Internet]. The Worldwide Hospice Palliative Care Alliance. World Hospice Palliative Care Alliance; [cited 2020 Mar 10]. Available online: http://www.thewhpca.org/resources/ category/standards-clinical-guidelines-and-protocols

14. Global Atlas of Palliative Care at the End of Life [Internet]. 0020Global_Atlas_of_Palliative_Care.pdf. Worldwide Palliative Care Alliance; 2014 [cited 2020 Feb 20]. Available online: https://www.who.int/nmh/Global_Atlas_ of_Palliative_Care.pdf

15. Clark D, Wright $M$. The international observatory on end of life care: a global view of palliative care development. J Pain Symptom Manage 2007;33:542-6.

16. Kirov L. Palliative care in Bulgaria: Structure and Human Site - both sides of the chain. M Silberman Palliative care reports from developing countries. 2019;3:27-36. ISBN 9781-53616-211-0, Nova Science Publishers, Inc. New York.
17. Political declaration of the high-level meeting on universal health coverage [Internet]. A/RES/74/2. United Nations General Assembly; 2019 [cited 2020 Feb 16]. Available online: https://undocs.org/en/A/RES/74/2

18. Panke JT, Ferrell BR. The family perspective. In: Hanks G, Cherny N, Christakis NA, Fallon M, Kaasa S, Portenoy RK, eds. Oxford Textbook of Palliative Medicine. 4th ed. Oxford: Oxford University Press; 2010:1437-44.

19. Palliative Care Definition [Internet]. IAHPC. International Association for Hospice \& Palliative Care; 2018 [cited 2020 Feb 22]. Available online: https://hospicecare.com/ what-we-do/projects/consensus-based-definition-ofpalliative-care/definition/

20. Steele R, Davies B. Supporting Families in Palliative Care - Oxford Medicine [Internet]. Supporting Families in Palliative Care - Oxford Medicine. Oxford University Press; 2017 [cited 2020 Feb 21]. Available online: https://oxfordmedicine.com/view/10.1093/ med/9780190244132.001.0001/med-9780190244132chapter-3

21. Murray MA, Fiset V, Young S, et al. Where the dying live: a systematic review of determinants of place of end-of-life cancer care. Oncol Nurs Forum 2009;36:69-77.

22. Gomes B, Calanzani N, Gysels M, et al. Heterogeneity and changes in preferences for dying at home: a systematic review. BMC Palliat Care 2013;12:7.

23. Higginson IJ, Sen-Gupta GJ. Place of care in advanced cancer: a qualitative systematic literature review of patient preferences. J Palliat Med 2000;3:287-300.

24. Norman GJ, Orton K, Wade A, et al. Operation and challenges of home-based medical practices in the US: findings from six aggregated case studies. BMC Health Serv Res 2018;18:45.

25. Atreya S, Patil C, Kumar R. Integrated primary palliative care model; facilitators and challenges of primary care/ family physicians providing community-based palliative care. J Family Med Prim Care 2019;8:2877-81.

26. Rustagi N, Pareek P, Verma $M$, et al. Essential Yet Neglected Role of Primary Care Physicians in Palliation Team. J Assoc Physicians India 2019;67:81-3.

27. Calton BA, Thompson N, Shepard N, et al "She Would Be Flailing Around Distressed": The Critical Role of Home-Based Palliative Care for Patients with Advanced Cancer. J Palliat Med 2017;20:875-8.

28. Peng JK, Chiu TY, Hu WY, et al. What influences the willingness of community physicians to provide palliative care for patients with terminal cancer? Evidence from a nationwide survey. Jpn J Clin Oncol 2013;43:278-85. 
29. Peng JK, Hu WY, Hung SH, et al. What can family physicians contribute in palliative home care in Taiwan? Fam Pract 2009;26:287-93.

30. Rahman AN, Rahman M. Home-Based Palliative Care: Toward a Balanced Care Design. J Palliat Med 2019;22:1274-80.

31. Rotter B, Grant M. Symptom Assessment and Hospital Utilization in a Home-Based Palliative Care Program. J Hosp Palliat Nurs 2018;20:332-7.

32. Cai J, Guerriere DN, Zhao H, et al. Correlation of Palliative Performance Scale and Survival in Patients With Cancer Receiving Home-Based Palliative Care. J Palliat Care 2018;33:95-9.

33. Nguyen HQ, Mularski RA, Edwards PE, et al. Protocol for a Noninferiority Comparative Effectiveness Trial of Home-Based Palliative Care (HomePal). J Palliat Med 2019;22:20-33.

34. Garrouste-Orgeas M, Flahault C, Poulain E, et al. The Fami-life study: protocol of a prospective observational multicenter mixed study of psychological consequences of grieving relatives in French palliative care units on behalf of the family research in palliative care (F.R.I.P.C research network). BMC Palliat Care 2019;18:111.

35. Sampson EL, Anderson JE, Candy B, et al. Empowering Better End-of-Life Dementia Care (EMBED-Care): A mixed methods protocol to achieve integrated personcentred care across settings. Int J Geriatr Psychiatry 2020;35:820-32.

36. Postier A, Catrine K, Remke S. Interdisciplinary Pediatric Palliative Care Team Involvement in Compassionate Extubation at Home: From Shared Decision-Making to Bereavement. Children (Basel) 2018;5:37.

37. Johnson CE, Girgis A, Paul CL, et al. Palliative care referral practices and perceptions: the divide between metropolitan and non-metropolitan general practitioners. Palliat Support Care 2011;9:181-9.

38. Yuen KJ, Behrndt MM, Jacklyn C, et al. Palliative care at home: general practitioners working with palliative care teams. Med J Aust 2003;179:S38-40.

39. Danielsen BV, Sand AM, Rosland JH, et al. Experiences and challenges of home care nurses and general practitioners in home-based palliative care - a qualitative study. BMC Palliat Care 2018;17:95.

40. Grinman MN, Human T, Shankel MJ, et al. Keeping Long-Term Care Patients Out of Hospital During Acute Medical Illness: Proposal for Common Elements of an Integrated Healthcare Delivery System for Long-Term Care. Healthc Q 2019;22:40-6.
41. Heath I. A general practitioner for every person in the world. BMJ 2008;336:861.

42. Fernandes R, Braun KL, Ozawa J, et al. Home-based palliative care services for underserved populations. J Palliat Med 2010;13:413-9.

43. Brzoska P, Yilmaz-Aslan Y, Probst S. Considering diversity in nursing and palliative care - the example of migrants. $Z$ Gerontol Geriatr 2018;51:636-41.

44. Jansky M, Owusu-Boakye S, Nauck F. "An odyssey without receiving proper care" - experts' views on palliative care provision for patients with migration background in Germany. BMC Palliat Care 2019;18:8.

45. Teunissen E, Gravenhorst K, Dowrick C, et al. Implementing guidelines and training initiatives to improve cross-cultural communication in primary care consultations: a qualitative participatory European study. Int J Equity Health 2017;16:32.

46. Boucher NA, Johnson KS. Cultivating Cultural Competence: How Are Hospice Staff Being Educated to Engage Racially and Ethnically Diverse Patients? Am J Hosp Palliat Care 2021;38:169-74.

47. Jansky M, Owusu-Boakye S, Nauck F. Palliative care for patients with Turkish or Arabic migration background in Lower Saxony: A survey from palliative care professionals' perspective. Bundesgesundheitsblatt Gesundheitsforschung Gesundheitsschutz 2017;60:45-54.

48. Shabnam J, Timm H, Nielsen DS, et al. Palliative care for older South Asian migrants: A systematic review. Palliat Support Care 2020;18:346-58.

49. Wales J, Isenberg SR, Wegier P, et al. Providing Medical Assistance in Dying within a Home Palliative Care Program in Toronto, Canada: An Observational Study of the First Year of Experience. J Palliat Med 2018;21:1573-9.

50. Pinderhughes ST, Lehn JM, Kamal AH, et al. Expanding Palliative Medicine across Care Settings: One Health System Experience. J Palliat Med 2018;21:1272-7.

51. Ajjarapu AS, Broderick A. Home-Based Palliative Care Program Relieves Chronic Pain in Kerala, India: Success Realized Through Patient, Family Narratives. Perm J 2018;22:17-151.

52. Ankuda CK, Kersting K, Guetterman TC, et al. What Matters Most? A Mixed Methods Study of Critical Aspects of a Home-Based Palliative Program. Am J Hosp Palliat Care 2018;35:236-43.

53. Seow H, Bainbridge D. The development of specialized palliative care in the community: A qualitative study of the evolution of 15 teams. Palliat Med 2018;32:1255-66. 
54. Sheehan OC, Ritchie CS, Fathi R, et al. Development of Quality Indicators to Address Abuse and Neglect in Home-Based Primary Care and Palliative Care. J Am Geriatr Soc 2016;64:2577-84.

55. Brian Cassel J, Kerr KM, McClish DK, et al. Effect of a Home-Based Palliative Care Program on Healthcare Use and Costs. J Am Geriatr Soc 2016;64:2288-95.

56. Palmer JA, Smith AM, Paasche-Orlow RS, et al. Research Literature on the Intersection of Dementia, Spirituality, and Palliative Care: A Scoping Review. J Pain Symptom Manage 2020;60:116-34.

57. Bowman BA, Twohig JS, Meier DE. Overcoming Barriers to Growth in Home-Based Palliative Care. J Palliat Med 2019;22:408-12.

58. Golden AG, Antoni C, Gammonley D. A Home-Based Palliative Care Consult Service for Veterans. Am J Hosp Palliat Care 2016;33:858-62.

59. Chandrashekar P, Moodley S, Jain SH. 5 Obstacles to home-based health care, and how to overcome them [Internet]. Harvard Business Review 2019 [cited 2020Mar5]. Available online: https://hbr.org/2019/10/5obstacles-to-home-based-health-care-and-how-toovercome-them

60. DeMiglio L, Williams A. Shared care: the barriers encountered by community-based palliative care teams in Ontario, Canada. Health Soc Care Community 2012;20:420-9.

61. O'Malley K, Blakley L, Ramos K, et al. Mental healthcare and palliative care: barriers. BMJ Support Palliat Care 2021;11:138-44.

62. Walter JK, Hill DL, DiDomenico C, et al. A conceptual model of barriers and facilitators to primary clinical teams requesting pediatric palliative care consultation based upon a narrative review. BMC Palliat Care 2019;18:116.

63. Vanderhaeghen B, Bossuyt I, De Nys K, et al. 'We need a physician who is a human being too': exploration of barriers and facilitators for hospitalised palliative patients and their families to discuss advance care planning. Int J Palliat Nurs 2019;25:603-9.

64. Mathew C, Hsu AT, Prentice M, et al. Economic evaluations of palliative care models: A systematic review. Palliat Med 2020;34:69-82.

65. Chen CY, Naessens JM, Takahashi PY, et al. Improving Value of Care for Older Adults With Advanced Medical Illness and Functional Decline: Cost Analyses of a HomeBased Palliative Care Program. J Pain Symptom Manage 2018;56:928-35.

66. Official final version prepared for WONCA-Region
Europe Conference in KOS, Greece 2005 [Internet]. Euract def. The EURACT Educational Agenda of General Practice/Family Medicine; 2005 [cited 2020Feb18]. Available online: https://euract.woncaeurope.org/ sites/euractdev/files/documents/publications/officialdocuments/euract-educationalagenda.pdf

67. Reckrey JM, Ornstein KA, Wajnberg A, et al. Teaching Home-Based Primary Care. Home Healthc Now 2017;35:561-5.

68. Reckrey JM, Willner MJ, DeCherrie LV, et al. HomeBased Primary Care as a Teaching Site for Palliative Care. J Palliat Med 2020;23:7.

69. Billings JA, Ferris FD, Macdonald N, et al. The role of palliative care in the home in medical education: report from a national consensus Conference. J Palliat Med 2001;4:361-71.

70. Langer T, Martinez W, Browning DM, et al. Patients and families as teachers: a mixed methods assessment of a collaborative learning model for medical error disclosure and prevention. BMJ Qual Saf 2016;25:615-25.

71. Aires MJ, Gagnayre R, Gross O, et al. The Patient Teacher in General Practice Training: Perspectives of Residents. J Patient Exp 2019;6:287-95.

72. Wakefield MA, Beilby J, Ashby MA. General practitioners and palliative care. Palliat Med 1993;7:117-26.

73. Boakes J, Gardner D, Yuen K, et al. General practitioner training in palliative care: an experiential approach. J Palliat Care 2000;16:11-9.

74. von Gunten CF, Neely KJ, Martinez J. Hospice and palliative care: program needs and academic issues. Oncology (Williston Park) 1996;10:1070-4; discussion 1074, 1079-80.

75. Klein S, Hostetter M, McCarthy D. An Overview of Home-Based Primary Care: Learning from the Field. Issue Brief (Commonw Fund) 2017;15:1-20.

76. Naicker SN, Richter L, Stein A, et al. Development and pilot evaluation of a home-based palliative care training and support package for young children in southern Africa. BMC Palliat Care 2016;15:41.

77. Caswell G, Hardy B, Ewing G, et al. Supporting family carers in home-based end-of-life care: using participatory action research to develop a training programme for support workers and volunteers. BMJ Support Palliat Care 2019;9:e4.

78. Despotova-Toleva L. Palliative care in childhood 2004, Medical Publishing House VAP, ISBN 954-9806-51-0.

79. Despotova-Toleva L. Education and training for palliative care purpose, In ed. A. Atanasov, Fundamentals of 
Palliative Medicine, 2005:160-70.

80. Despotova-Toleva L. Scientific/research aspect of palliative medicine. In ed. A. Atanasov, Fundamentals of Palliative Medicine, 2005:171-4.

81. Despotova-Toleva L. Juridical aspect of palliative medicine in ed. A. Atanasov, Fundamentals of Palliative Medicine, 2005:175-8.

82. Despotova-Toleva L. Financial and social aspect of palliative medicine in ed. A. Atanasov, Fundamentals of Palliative Medicine, 2005:179-83.

83. Despotova-Toleva L. Modern ICT in palliative medicine in ed. A. Atanasov, Fundamentals of Palliative Medicine, 2005:184-92.

84. Despotova-Toleva L. Home care for palliative patients in ed. A. Atanasov, Fundamentals of Palliative Medicine, 2005:193-202.

85. Despotova-Toleva L, Yordanov N. Practicals in Long term and Palliative care, Lax Book, 2017.

86. Working group, Medical Standard for Palliative Care, Folia Palliatrica 1, 2017; 12-36 Available online: https:// palliamed.org/wp-content/plugins/pdfjs-viewershortcode/pdfjs/web/viewer.php?file=https $\% 3 \mathrm{~A} \% 2 \mathrm{~F} \% 2 \mathrm{Fp}$ alliamed.org\%2Fwp-content \%2Fuploads\%2F2017\%2F04 \%2FFolia_Palliatrica_vol_I_cover_ed_page.pdf\&downloa $\mathrm{d}=$ true\&print=true\&openfile=false

87. Trollor J. Rural general practitioners and palliative care in the north-west of New South Wales. Aust Fam Physician 1995;24:1106-9, 1112-3.

88. Evans R, Stone D, Elwyn G. Organizing palliative care for rural populations: a systematic review of the evidence. Fam Pract 2003;20:304-10.

89. Robinson CA, Pesut B, Bottorff JL. Supporting rural family palliative caregivers. J Fam Nurs 2012;18:467-90.

90. Piamjariyakul U, Petitte T, Smothers A, et al. Study protocol of coaching end-of-life palliative care for advanced heart failure patients and their family caregivers in rural appalachia: a randomized controlled trial. BMC Palliat Care 2019;18:119.

91. Marston J, Boucher S, Downing J. International Children's Palliative Care Network: A Global Action Network for Children With Life-Limiting Conditions. J Pain Symptom Manage 2018;55:S104-S111.

92. De Lima L, Radbruch L. The International Association for Hospice and Palliative Care: Advancing Hospice and Palliative Care Worldwide. J Pain Symptom Manage 2018;55:S96-S103.

93. Cottrell L, Duggleby W. The "good death": An integrative literature review. Palliat Support Care 2016;14:686-712.
94. Gomes B, Calanzani N, Curiale V, et al. Effectiveness and cost-effectiveness of home palliative care services for adults with advanced illness and their caregivers. Cochrane Database Syst Rev 2013;(6):CD007760.

95. Higginson IJ, Sarmento VP, Calanzani N, et al. Dying at home--is it better: a narrative appraisal of the state of the science. Palliat Med 2013;27:918-24.

96. Abarshi E, Onwuteaka-Philipsen B, Donker G, et al. General practitioner awareness of preferred place of death and correlates of dying in a preferred place: a nationwide mortality follow-back study in the Netherlands. J Pain Symptom Manage 2009;38:568-77.

97. Meeussen K, Van den Block L, Bossuyt N, et al. GPs' awareness of patients' preference for place of death. Br J Gen Pract 2009;59:665-70.

98. Walsh D, Regan J. Terminal care in the home--the general practice perspective. Ir Med J 2001;94:9-11.

99. De Roo ML, Miccinesi G, Onwuteaka-Philipsen BD, et al. EURO IMPACT. Actual and preferred place of death of home-dwelling patients in four European countries: making sense of quality indicators. PLoS One 2014;9:e93762.

100. Ko W, Miccinesi G, Beccaro M, et al. Factors associated with fulfilling the preference for dying at home among cancer patients: the role of general practitioners. J Palliat Care 2014;30:141-50.

101.Ko W, Beccaro M, Miccinesi G, et al. Awareness of general practitioners concerning cancer patients' preferences for place of death: evidence from four European countries. Eur J Cancer 2013;49:1967-74.

102.Higginson IJ, Daveson BA, Morrison RS, et al. Social and clinical determinants of preferences and their achievement at the end of life: prospective cohort study of older adults receiving palliative care in three countries. BMC Geriatr 2017;17:271.

103. Skorstengaard MH, Neergaard MA, Andreassen P, et al. Preferred Place of Care and Death in Terminally Ill Patients with Lung and Heart Disease Compared to Cancer Patients. J Palliat Med 2017;20:1217-24.

104. Rainsford S, MacLeod RD, Glasgow NJ. Place of death in rural palliative care: A systematic review. Palliat Med 2016;30:745-63.

105. Chukwusa E, Verne J, Polato G, et al. Urban and rural differences in geographical accessibility to inpatient palliative and end-of-life (PEoLC) facilities and place of death: a national population-based study in England, UK. Int J Health Geogr 2019;18:8.

106. Dong T, Zhu Z, Guo M, et al. Association between 
Dying Experience and Place of Death: Urban-Rural Differences among Older Chinese Adults. J Palliat Med 2019;22:1386-93.

107.Johnson CE, McVey P, Rhee JJ, et al. General practice palliative care: patient and carer expectations, advance care plans and place of death-a systematic review. BMJ Support Palliat Care 2018. doi: 10.1136/bmjspcare-2018-001549.

108. Martoni AA, Strocchi E. Accessibility of home palliative care and place of death of cancer patients: data analysis of the Emilia-Romagna Region. Recenti Prog Med 2018;109:185-92.

109. Barclay S, Wyatt P, Shore S, et al. Caring for the dying: how well prepared are general practitioners? A questionnaire study in Wales. Palliat Med 2003;17:27-39.

110. Sirintrapun SJ, Lopez AM. Telemedicine in Cancer Care. Am Soc Clin Oncol Educ Book 2018;38:540-5.

111. Hebert MA, Paquin MJ, Whitten L, et al. Analysis of the suitability of 'video-visits' for palliative home care: implications for practice. J Telemed Telecare 2007;13:74-8.

112. Kidd L, Cayless S, Johnston B, et al. Telehealth in palliative care in the UK: a review of the evidence. $J$ Telemed Telecare 2010;16:394-402.

113. Duursma F, Schers HJ, Vissers KC, et al. Study protocol: optimization of complex palliative care at home via telemedicine. A cluster randomized controlled trial. BMC Palliat Care 2011;10:13.

114. Stern A, Valaitis R, Weir R, et al. Use of home telehealth in palliative cancer care: a case study. J Telemed Telecare 2012;18:297-300

115. Hoek PD, Schers HJ, Bronkhorst EM, et al. The effect of weekly specialist palliative care teleconsultations in patients with advanced cancer -a randomized clinical trial. BMC Med 2017;15:119.

116. Bradford NK, Young J, Armfield NR, et al. Home telehealth and paediatric palliative care: clinician perceptions of what is stopping us? BMC Palliat Care 2014;13:29.

117.Pinto S, Caldeira S, Martins JC. e-Health in palliative care: review of literature, Google Play and App Store. Int J Palliat Nurs 2017;23:394-401.

118. Meghani SH, MacKenzie MA, Morgan B, et al. ClinicianTargeted Mobile Apps in Palliative Care: A Systematic Review. J Palliat Med 2017;20:1139-47.

119. Freire de Castro Silva SL, Gonçalves AA, Cheng C, et al. Mobile Technology Applications in Cancer Palliative Care. Stud Health Technol Inform 2018;249:199-202.

120.Allsop MJ, Namisango E, Powell RA. A survey of mobile phone use in the provision of palliative care services in the
African region and priorities for future development. J

Telemed Telecare 2019;25:230-40.

121. Lindley LC, Zhou W, Mack JW, et al. Pediatric hospice and palliative care: designing a mobile app for clinical practice. Comput Inform Nurs 2014;32:299-302.

122. Bienfait F, Petit M, Pardenaud R, et al. Applying M-Health to Palliative Care: A Systematic Review on the Use of M-Health in Monitoring Patients With Chronic Diseases and its Transposition in Palliative Care. Am J Hosp Palliat Care 2020;37:549-64.

123. Dickman Portz J, Ford K, Bekelman DB, et al. "We're Taking Something So Human and Trying to Digitize": Provider Recommendations for mHealth in Palliative Care. J Palliat Med 2020;23:240-7.

124. Salimian N, Ehteshami A, Ashouri-Talouki M. Developing Ghasedak: a Mobile Application to Improve the Quality of Cancer Palliative Care. Acta Inform Med 2019;27:19-22.

125. Flint R, Buchanan D, Jamieson S, et al. The Safer Prescription of Opioids Tool (SPOT): A Novel Clinical Decision Support Digital Health Platform for Opioid Conversion in Palliative and End of Life Care-A SingleCentre Pilot Study. Int J Environ Res Public Health 2019;16:1926.

126. Doolittle GC, Nelson EL, Spaulding AO, et al. TeleHospice: A Community-Engaged Model for Utilizing Mobile Tablets to Enhance Rural Hospice Care. Am J Hosp Palliat Care 2019;36:795-800.

127.Pinto S, Almeida F, Caldeira S, et al. The Comfort app prototype: introducing a web-based application for monitoring comfort in palliative care. Int J Palliat Nurs 2017;23:420-31.

128. Weekly T, Walker N, Beck J, et al. A Review of Apps for Calming, Relaxation, and Mindfulness Interventions for Pediatric Palliative Care Patients. Children (Basel) 2018;5:16.

129.Lehto RH, Heeter C, Allbritton M, et al. Hospice and Palliative Care Provider Experiences With Meditation Using Mobile Applications. Oncol Nurs Forum 2018;45:380-8.

130. Millares Martin P. Electronic palliative care coordination system (EPaCCS) in practice: A useful tool? BMJ Support Palliat Care 2021;11:146-8.

131.Hancock S, Preston N, Jones H, et al. Telehealth in palliative care is being described but not evaluated: a systematic review. BMC Palliat Care 2019;18:114.

132. Sanderson C, Tieman J. CareSearch - online palliative care information for GPs. Aust Fam Physician 2010;39:341-3.

133. Funderskov KF, Boe Danbjørg D, Jess M, et 
al. Telemedicine in specialised palliative care:

Healthcare professionals' and their perspectives on video consultations-A qualitative study. J Clin Nurs 2019;28:3966-76.

134. Funderskov KF, Raunkiær M, Danbjørg DB, et al. Experiences With Video Consultations in Specialized Palliative Home-Care: Qualitative Study of Patient and Relative Perspectives. J Med Internet Res 2019;21:e10208. 135. Neergaard MA, Warfvinge JE, Jespersen TW, et al. The impact of "modern telecommunication" in palliative care--the views of the professionals. Telemed J E Health 2014;20:24-31.

136.van Gurp J, Hasselaar J, van Leeuwen E, et al. Connecting with patients and instilling realism in an era of emerging

Cite this article as: Despotova-Toleva L, Toleva-Nowak N. Narrative review of home care for palliative patients in general practice. Ann Palliat Med 2021;10(12):13009-13023. doi: 10.21037/ apm-2021-01 communication possibilities: a review on palliative care communication heading to telecare practice. Patient Educ Couns 2013;93:504-14.

137.van Gurp J, van Selm M, Vissers K, et al. How outpatient palliative care teleconsultation facilitates empathic patientprofessional relationships: a qualitative study. PLoS One 2015;10:e0124387.

138. Bradford N, Young J, Armfield NR, et al. A pilot study of the effectiveness of home teleconsultations in paediatric palliative care. J Telemed Telecare 2012;18:438-42.

139. Bradford NK, Armfield NR, Young J, et al. Principles of a paediatric palliative care consultation can be achieved with home telemedicine. J Telemed Telecare 2014;20:360-4. 